\title{
Kostenloser ÖPNV? Besser gar nicht als falsch einführen
}

Will man mit Bussen, Straßen- oder U- und S-Bahnen in den Städten (im Folgenden ungenau als ÖPNV bezeichnet) Passagiere transportieren, so muss man die Strecken, die Haltepunkte, die Fahrzeiten und die Preise festlegen. Zum besseren Verständnis der optimalen Bepreisung hilft eine Marginalbetrachtung: In einer erstbesten Welt würde der Preis für einen Passagier so hoch sein wie die zusätzlichen Kosten, die seine Mitfahrt verursacht. Fährt der Bus fast leer, so verursacht die Mitfahrt sehr geringe Kosten: Der Sitz wird etwas schneller abgenutzt und der Motor verbrennt ein wenig mehr Treibstoff. Ein- und Aussteigen jedoch kostet Zeit, und wenn dieses Verspätungen verursacht, sind die Mitfahrer negativ betroffen; und zwar die, die schon an Bord sind und länger fahren sowie die, die später auf der Route noch einsteigen wollen und zu früh zur Bushaltestelle gekommen sind. Optimal sind sehr geringe Preise, die nur für das Ein- und Aussteigen verlangt werden und nicht mit der Distanz ansteigen, in der Regel sogar Preise von Null.

Während der Stoßzeit jedoch ändert sich das Bild. Jeder Passagier verschlechtert das Fahrerlebnis der anderen. Findet ein Passagier einen Sitzplatz, muss ein anderer stehen. Steht ein Passagier und kann sich gut festhalten, muss ein anderer ohne vernünftige Festhaltemöglichkeit mitfahren. Versperrt ein Passagier den Ausgang, verzögert sich das Ein- und Aussteigen und die Haltezeit des Fahrzeugs erhöht sich. Für die Bepreisung wichtig ist nicht, dass das eigene Fahrerlebnis (interne Kosten) eines Passagiers schlecht ist, sondern dass der Fahrkomfort der Mitfahrer (externe Kosten) durch ihn oder sie verschlechtert wird. Der optimale Preis umfasst alle diese externen Kosten. Er ist zusätzlich zur festen Komponente für das Ein- und Aussteigen distanzabhängig, weil der Komfortverlust sich über die Entfernung (oder Dauer) aggregiert, und er fällt für alle Teile der Route an, in denen der Besetzungsgrad des Fahrzeugs eine bestimmte Grenze überschreitet. Die optimale Kapazität des ÖPNV-Angebots minimiert die Summe aus Erstellungskosten, Zeitkosten und Komfortverlusten durch Überfüllung. Bietet man eine optimale Kapazität an, liegen konstante Kapazitätsgrenzkosten vor, und werden auch die Substitute (private Pkw, Taxi, Fahrrad, zu Fuß gehen) optimal bepreist, dann kann sich ein solches System vollständig selbst finanzieren. Dieses System enthält nur zu den Nebenverkehrszeiten eine entgeltfreie Nutzung, jedoch distanzabhängige Preise für die Hauptverkehrszeiten, jeweils gemessen am Besetzungsgrad bei optimaler Kapazität. Dabei wird auch betrachtet, dass mehr Kunden eine erhöhte Fahrtfrequenz erlauben, was sinkende Wartezeiten zur Folge hat (Mohring-Effekt).

Nun entstehen dem innerstädtischen Pkw-Verkehr zwar Grenzkosten des Treibstoffverbrauchs, keine jedoch für die verursachte Stauexternalität oder für emittierte Luftschadstoffe. Da auch nicht zu erwarten ist, dass es eine solche distanz- und belastungsabhängige Mauttechnologie in den nächsten Jahren zu akzeptablen Kosten gibt, ist für den ÖPNV nur eine zweitbeste Lösung möglich: Da die Grenzkosten für Pkw zu niedrig sind, sollten auch die Fahrpreise im ÖPNV niedriger als in einer erstbesten Welt gewählt werden. Gerade dies geschieht teilweise in Deutschland. Die Fahrpreiserlöse erreichen ungefähr nur drei Viertel der Kosten. Jedoch führt die auch im ÖPNV nur wenig ausgeprägte Preisdifferenzierung zwischen Haupt- und Nebenzeiten zur überhöhten Nutzung der Hauptzeiten, was die Attraktivität des ÖPNV für den Noch-Pkw-Fahrer verringert. Existieren hohe Zeitkosten bei den Pkw-Fahrern und ist die Stausituation extrem, wie z.B. in London, so beinhaltet eine zweitbeste Politik einen ÖPNV-Nulltarif selbst zur Stoßzeit, verbunden mit einem Ausbau der Kapazitäten. Werden die Kapazitäten nicht ausgebaut und verursachen Subventionen

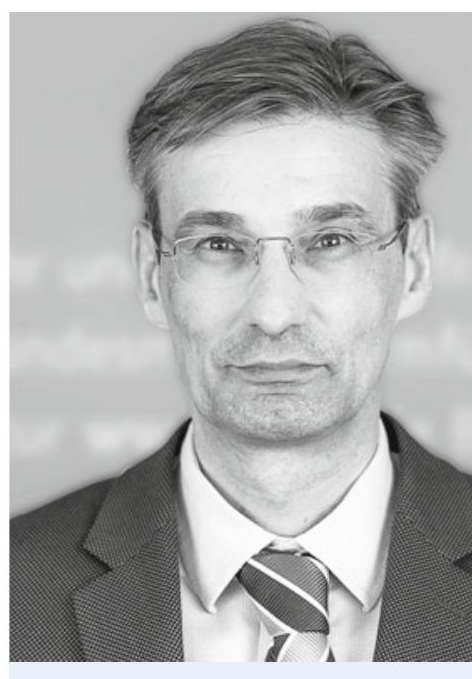

Gernot Sieg ist Direktor des Instituts für Verkehrswissenschaft an der Westfälischen WilhelmsUniversität Münster. 
aufgrund von ineffizienter Besteuerung Wohlfahrtsverluste, so würde jedoch auch im Beispiel London die Einführung von Nulltarifen zu einem Wohlfahrtsverlust führen. Die erstbeste Lösung in Bezug auf den Pkw-Verkehr ist immer die distanz- und belastungsabhängige City-Maut. Mit einer solchen Maut würde der wesentliche Grund für eine Subventionierung des ÖPNV wegfallen. Aus Gründen der Akzeptanz wird jedoch häufig empfohlen, etwaige City-Maut-Einnahmen zur Verbesserung des ÖPNV zu verwenden. Der öffentliche Verkehr ist insbesondere für Personen mit geringem Einkommen oft die einzige Möglichkeiten, längere Distanzen zu überwinden. Aufgrund von verteilungspolitischen Zielen und zur Absicherung der Teilhabe können und werden für solche Personen besonders subventionierte Fahrscheine angeboten.

Der Vorschlag der Bundesregierung, vorübergehend in fünf „lead cities“ „public transport free of charge" anzubieten, ist jedoch motiviert durch das EU-Vertragsverletzungsverfahren hinsichtlich von Luftschadstoffen, und hier insbesondere der Überschreitung der Jahresgrenzwerte bei Stickstoffoxiden in vielen deutschen Städten. Der gesunkene Fahrpreis im ÖPNV soll Pkw-Fahrer zum Umsteigen motivieren. Die empirischen Erfahrungen zeigen, dass dieses gelingen kann, aber nur in geringem Maße. Die Kostenvorteile des Massenpassagiertransports werden mit Nachteilen im Komfort, der Reisedauer und der Passung von gewünschten Abfahrts- und Ankunftszeiten erkauft. Die vollen Kosten der Fahrt bestehen nur zum Teil aus den Fahrscheinkosten, zum großen Teil auch aus Zeitkosten und Terminplanveränderungskosten. Von daher reagiert die ÖPNV-Nachfrage nur unelastisch auf Preisänderungen.

Insbesondere Dieselmotoren mit Schadstoffklassen, die schlechter als EURO-6d-temp sind, emittieren viel Stickstoffoxide. Ein Großteil der neuen Fahrgäste im ÖPNV jedoch fuhr vorher einen Benzin-Pkw, benutzte das Fahrrad oder ging zu Fuß. Die ÖPNV-Kapazitäten würden nicht ausreichen, sondern müssten erhöht werden. Der Prozess der Fahrplanentwicklung, der Beschaffung von Fahrzeugen und Fahrern würde mehrere Jahre dauern, der positive Effekt für die Luftschadstoffe also erst verzögert einsetzen. Der zusätzliche Subventionsbedarf würde zum einen die heutigen Fahrpreiserlöse weit übersteigen. Zum anderen wären die erzielten Effekte überwiegend Mitnahmeeffekte. Auch induziert der reduzierte Straßenverkehrsstau neuen Pkw-Verkehr.

Eine vollständige Subventionierung des ÖPNV durch den Staat verursacht Governance-Probleme bei der Bereitstellung der Leistungen. Informationen, welchen Wert die Transportdienstleistung hat, werden nicht mehr erfasst. Anreize, eine optimale Kapazität anzubieten, verschwinden. Der Umfang der Subventionierung und die Art der Subventionierung (staatliches Unternehmen, Ausschreibungswettbewerb) beeinflussen die Effizienz der Busunternehmen. Während der Streckenverlauf im Busverkehr flexibler ist und die Frequenz einfacher angepasst werden kann, ist dieses bei Straßen-, U-, und Schwebebahnen nur schwierig möglich. Eine schwer zu ändernde Anbindung an das ÖPNV-Netz (U-Bahn-Haltestelle) erhöht den Bodenwert in der Umgebung. Aus polit-ökonomischer Sicht ist also mit einer Überversorgung an schienengebundenem ÖPNV zu rechnen, die durch eine Subventionierung verschärft wird.

Für das Problem der Bundesregierung im EU-Vertragsverletzungsverfahren zu Luftschadstoffen in Städten ist der „public transport free of charge“ eine ineffiziente und wenig erfolgversprechende Politik. Kostenloser ÖPNV verschlingt immense Subventionen. Mit einem Bruchteil der Mittel könnte man sowohl die Luftreinheit in den Städten effektiv verbessern als auch positive Wohlfahrtswirkungen des ÖPNV an-

Gernot Sieg

Westfälische Wilhelms-

Universität Münster

gernot.sieg@uni-muenster.de stoßen. Eine bessere Preisdifferenzierung zwischen Neben- und Hauptverkehrszeiten und eine (höher als heute) subventionierte Verbesserung des Angebots zu den Hauptverkehrszeiten wäre in staugeplagten Städten ohne City-Maut die bessere Alternative. 